\title{
Expert S-plane Control of Underwater Vehicle
}

\section{Chunmeng Jiang1, Lei Wan2 \& Yushan Sun3}

1 State Key Laboratory of Autonomous Underwater Vehicle, Harbin Engineering University, Harbin, 150001, China.

2 State Key Laboratory of Autonomous Underwater Vehicle, Harbin Engineering University, Harbin, 150001, China.

3 State Key Laboratory of Autonomous Underwater Vehicle, Harbin Engineering University, Harbin, 150001, China

Keywords: Underwater vehicle(UV), Expert control, Intelligent integral.

Abstract. Study of motion control of underwater vehicle is deeply undertaken here. S-plane control is verified to be effective and validity in the control of UVs, but there are still problems in steady precision and parameter adjustments. To obtain higher steady precision, intelligent integral method is brought in, and expert S-plane control is introduced to adjust the control parameters on-line. To prevent output jumping, fuzzy neural network is adopted to fit the production rules in knowledge base. Experiments are finished, and the results demonstrate that the expert S-plane controller has better robustness than S-plane controller.

\section{Introduction}

With the exploit of activities in deep sea, the application of underwater vehicle (UV) is widespread. UV is a high nonlinear system with strong coupling, and its surrounding environment is very complex, therefore, the design of its control system should take nonlinear problem into account, and it should possess good adaptability and robustness.

The S-plane control method presented in [1] combines fuzzy control ideology with structure of PD control, it not only simplified controller design, but also enhance nonlinear control effect. To improve the working performance of UV, [2] refers to error back propagation algorithm of neural network, and adds self-learning function for S-plane controller. [3] adopts immune generic algorithm to optimize control parameters of S-plane controller, in order to reduce the uncertainty within the design process.

The methods mentioned above verified by sea trials and good control effect was obtained, however, there also exists something to be improved. Take [2] for example, precise teacher signal is difficult to get; based on the requirements of hardware velocity and real-time performance, the method in [3] is difficult to put into practice. Because of the deep study of S-plane control, and plenty of experience is accumulated[4], therefore, expert technology[5] can be brought in to enhance the control performance.

\section{S-plane Control Model Based on Intelligent Integration}

The control model of common S-plane controller is as follows:

$$
u=2.0 /\left(1.0+e^{\left(-k_{1} e-k_{2} \dot{e}\right)}\right)-1.0+\Delta u
$$

Where, $e_{\text {and }} \dot{e}$ are the control inputs(bias and bias rate, with normalization), $u$ is the control output, here $u$ represents the expected force in each DOF(normalization), $k_{1}$ and $k_{2}$ are the control parameters, $\Delta u$ is the fix disturb force according to the adaptability, and fix disturb force can be eliminated by adjusting $\Delta u$. Actually, $\Delta u$ is an integration item with the expression $\Delta u=k_{3} \int e d t$.

Though common S-plane controller has good robustness performance, it is one type of PD controller actually. To improve stable accuracy, the integration function can be added to reduce 
stable bias. The selection of common integration parameter $k_{3}$ is not easy, if too big, the system vibration is coming, however, if too small, the integration function is not obvious. Moreover, the integration goes with the bias, it is easy to result in "integration saturation" phenomena.

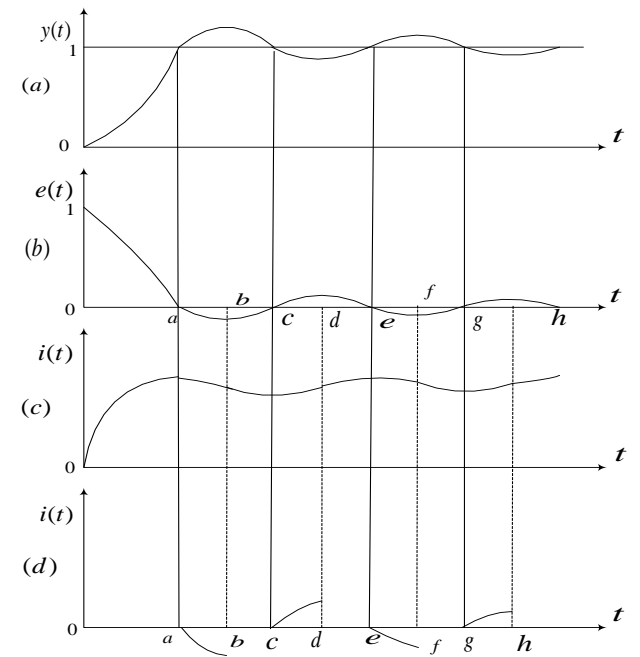

Fig. 1 Bias $e(\mathrm{t})$ and its integration curve $i(\mathrm{t})$

The reason result in the bad integration effect is it doesn't represent the control ideal of the skilful operators. In section [a, b] and [b, c] in fig.1(c), the integration function is against with the skilful operator's control effect. At this time, the system occurs overshot, the right control strategy should add a minus control effect to the control variable in order to reduce overshot. However, the integration function in this section adds a plus control effect, this is because the integration result can hardly counteract in section [0, a].

When refer to section [c, d], the plus control effect of integration effect beneficial to reduce output overshot. But section [d,e] doesn't meet this condition.

To overcome the disadvantage of the integration effect mentioned above, a new integration curve is adopted, namely, do integration in section [a, b]、 [c, d] and [e, f], this kind of integration can present right additional control item for the integration function in time, which can restrain the system error's increase; while stop integration in section $[0, a],[b, c]$ and $[d, e]$. At this time, the system is not belonging to out of control, as it is controlled by proportion control. This kind of integration imitates human's memory characteristic, and it selects to remember useful information, which possess apery intelligence, therefore, this kind of integration control is called apery intelligence integration control.

According to the analysis, the judgment condition which brought in integration is as follows: if $e \cdot \dot{e}>0$, then do integration, if $e \cdot \dot{e}<0$, then don't do integration. This is the basic condition for bringing in intelligent integration. Then think about the extreme point of bias and bias rate, the condition for bringing in intelligent integration can be concluded as follows:

If $e \cdot \dot{e}>0$ or $\dot{e}=0$ and $e \neq 0$, do integration,

If $e \cdot \dot{e}<0$ or $e=0$, don't do integration

The intelligent integration method can improve system's steady state precision. The S-plane control model contains intelligent integration is:

$$
\begin{aligned}
& u=2.0 /\left(1.0+e^{\left(-k_{1} e-k_{2} \dot{e}\right)}\right)-1.0+\Delta u, e \cdot \dot{e}>0 \text { or } \dot{e}=0 \text { and } e \neq 0 \\
& u=2.0 /\left(1.0+e^{\left(-k_{1} e-k_{2} \dot{e}\right)}\right)-1.0, e \cdot \dot{e}<0 \text { or } e=0
\end{aligned}
$$

\section{Expert Control}

The basic definition of expert control is "combine the theory and technology of expert system 
with controls, simulate experts' intelligence, and realize system's control in unknown condition [6]”.

At the beginning, we need to acquire enough knowledge from domain experts and skilful operators before the expert controller set up[7]. This knowledge will be sent into knowledge database for storage[8], the reasoning machine will call back the knowledge database's knowledge to reasoning, the knowledge after reasoning will be store in knowledge database and control rule set, and it will control the control plant[9]. The output of control plant feedback to information acquisition and processing unit as feedback information[10], the output of control plant will be compared with the setting value, and the steps will be repeated to realize real-time adjustment.

In common sense, the expert controller is made up of four parts, including information acquisition and processing, knowledge database, reasoning structure and control rule set.

\section{Expert S-plane Control}

From (2), we know that the S-plane control model with intelligent integration just has three control parameters, $k_{1} 、 k_{2}$ and $k_{3}$, the parameters need adjusting is greatly reduced compared with fuzzy control. The $k_{1}$ and $k_{2}$ can adjust the overshot and convergence speed of control, while $k_{3}$ can influence steady error.

The expert control proposed here is a kind of intelligent control which combines expert system technology and S-plane control. It adjust the $k_{1} 、 k_{2}$ and $k_{3}$ online based on the system performance, to make the system performance meet the requirement. The basic control adopts $\mathrm{S}$ plane controller; the expert intelligent harmonization level is made up of database(which store error threshold, error rate threshold, etc.), knowledge database and intelligent harmonization machine(reasoning machine). It will monitor the performance of control system online.

The influence of $k_{1}$ and $k_{2}$ in S-plane controller is as follows:

$k_{1}$ affect $e$. If we increase $k_{1}$, then the control function of $e$ is increased also, therefore, the rising time is shorten but the overshot is increased, and the convergence speed become slow, so, the $k_{1}$ should not choose too large. If we reduce $k_{1}$, the control function of $e$ is reduced, this is beneficial to reduce overshot, but if $k_{1}$ too small, the error play less important role, then the rising time become long, the steady state error increase, this is also not good for convergence. However, the parameter of $\boldsymbol{k}_{2}$ do the opposite thing.

Based on the analysis above, we know that $k_{1}$ and $k_{2}$ play different role in system's response, according to analysis above and the control system design experience, the fuzzy neural network can be used for approaching the rule in knowledge database, adjustment $k_{1}$ and $k_{2}$, we can avoid the jumping in control output.

Based on the analysis above and the control system design experience, we know that $k_{1}$ and $k_{2}$ play different role in system's response, and the fuzzy neural network can be used for approaching the rule in knowledge database, adjustment $k_{1}$ and $k_{2}$, we can avoid the jumping in control output. 


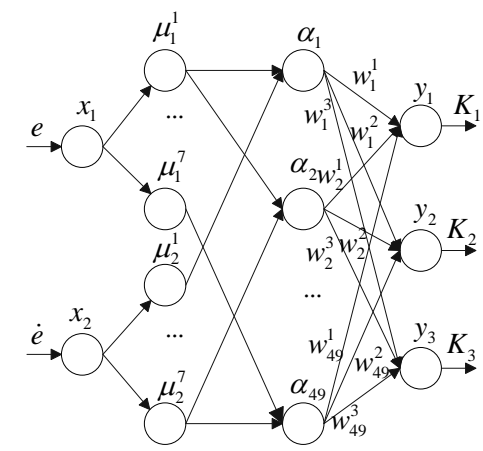

Fig.2 The structure of fuzzy neural network

The structure of fuzzy neural network is shown in Fig.2. The input is $e$ and $\dot{e}$, and the output is expected force or moment in each DOF. Where, the first layer is input layer, $x_{1}=e, x_{2}=\dot{e}$. The second layer is fuzzy layer, the corresponding $e$ and $\dot{e}$ has seven member function respectively, $\mu_{i}^{j}=\exp \left[-\frac{\left(x_{i}-m_{i j}\right)^{2}}{\sigma_{i j}{ }^{2}}\right], i=1,2, \ldots, 25, j=1,2,3$. Where, $i=1,2, j=1,2, \ldots, 5,$. The third layer is rule layer, $\alpha_{k}=\mu_{1}^{m} \cdot \mu_{2}^{n}, m=1,2, \ldots, 5, n=1,2, \ldots, 5$, therefore, there's 25 nodes in this layer. The fourth layer is output layer. $w_{i}^{j}$ is the control rule, $i=1,2, \ldots, 25, j=1,2,3$.

The function of this fuzzy neural network is equal to a fuzzy system[7],the reason why we choose neural network for expressing is the intuitionist character. The fuzzy language variables belong to fuzzy convene $\{\mathrm{NL}, \mathrm{NS}, \mathrm{ZE}, \mathrm{PS}, \mathrm{PL}\}$. The format of control rule ${ }^{w_{i}^{j}}$ is as follows:

Table 1: Rule $K_{1}$

\begin{tabular}{cccccc}
\hline$\dot{e} \backslash \mathrm{e}$ & $\mathrm{NL}$ & $\mathrm{NS}$ & $\mathrm{ZE}$ & $\mathrm{PS}$ & $\mathrm{PL}$ \\
\hline $\mathrm{NL}$ & 1.0 & 2.25 & 3.0 & 2.25 & 1.0 \\
$\mathrm{NS}$ & 0.25 & 1.75 & 2.25 & 1.75 & 0.25 \\
$\mathrm{ZE}$ & 0.0 & 1.25 & 2.0 & 1.25 & 0.0 \\
$\mathrm{PS}$ & 0.25 & 1.75 & 2.25 & 1.75 & 0.25 \\
$\mathrm{PL}$ & 1.0 & 2.25 & 3.0 & 2.25 & 1.0 \\
\hline \multicolumn{5}{c}{ Table 2: } & Rule $K_{2}$ \\
\hline$\dot{e} \backslash \mathrm{e}$ & $\mathrm{NL}$ & $\mathrm{NS}$ & $\mathrm{ZE}$ & $\mathrm{PS}$ & $\mathrm{PL}$ \\
\hline $\mathrm{NL}$ & 3.0 & 1.25 & 0.0 & 0.25 & 2.0 \\
$\mathrm{NS}$ & 2.75 & 1.0 & 0.0 & 0.75 & 2.25 \\
$\mathrm{ZE}$ & 2.5 & 0.8 & 0.0 & 0.8 & 2.5 \\
$\mathrm{PS}$ & 2.25 & 0.75 & 0.0 & 1.0 & 2.75 \\
$\mathrm{PL}$ & 2.0 & 0.25 & 0.0 & 1.25 & 3.0 \\
\hline \multicolumn{7}{c}{ Table 3: } & Rule $K_{3}$ & & \\
\hline$\dot{e} \backslash \mathrm{e}$ & $\mathrm{NL}$ & NS & ZE & PS & PL \\
\hline $\mathrm{NL}$ & 3.0 & 3.0 & 0.0 & 0.0 & 0.0 \\
$\mathrm{NS}$ & 3.0 & 3.0 & 0.0 & 0.0 & 0.0 \\
$\mathrm{ZE}$ & 3.0 & 3.0 & 0.0 & 3.0 & 3.0 \\
$\mathrm{PS}$ & 0.0 & 0.0 & 0.0 & 3.0 & 3.0 \\
$\mathrm{PL}$ & 0.0 & 0.0 & 0.0 & 3.0 & 3.0 \\
\hline
\end{tabular}

After setting up the knowledge database, $k_{1} 、 k_{2}$ and $k_{3}$ can be adjustment with (3): 


$$
\left\{\begin{array}{l}
k_{e}=\alpha+\lambda_{1} K_{1} \\
k_{v}=\beta+\lambda_{2} K_{2} \\
k_{i}=\gamma+\lambda_{3} K_{3}
\end{array}\right.
$$

Where, $\alpha, \beta, \gamma$ are the initial value for $k_{1} 、 k_{2}$ and $k_{3}$ respectively, $\alpha=\beta=3.0, \gamma=0$. $\lambda_{i}(i=1,2,3)$ is the restriction factor, $\lambda_{1}=0.15, \lambda_{2}=0.1, \lambda_{3}=0.05$.

\section{Simulation experiment}

Considering the velocity of the UV, the motion in each DOF is decoupling, the coupling effect between each DOF is simplified into outer disturbance. In order to obtain more precise motion model, the simulator's frequency use $40 \mathrm{~Hz}$, but in order to simulate, the simulate frequency of controller should choose $2 \mathrm{~Hz}$.

To verify the performance of water flow disturbance of expert S-plane controller, the positioning control experiment in water flow environment is undertaken. Fig.3 shows the response of longitudinal positioning control in water flow condition. The initial state of UV is 0 , and the target point is $5 \mathrm{~m}$ in longitudinal axis. The water flow velocity is $0.5 \mathrm{~m} / \mathrm{s}$, with the bearing angle of 180 degree. From fig.3, we know that expert S-plane controller achieve faster response speed, and the steady state precision gets better. The water flow resistance ability of expert S-plane controller is better than S-plane controller.

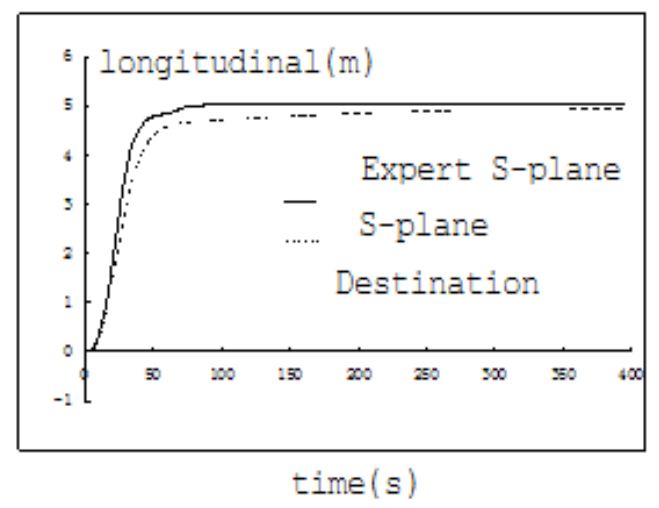

Fig.3 Longitudinal positioning control with sea current $0.5 \mathrm{~m} / \mathrm{s}$

\section{Conclusions}

This paper aims to improve the S-plane controller. The expert system technology is combined with S-plane controller, and overcome the real-time parameter adjustment in S-plane controller. The intelligent integration is brought in, and the steady state precision is enhanced effectively. Based on the knowledge database of fuzzy neural network, the disadvantages of fuzzy rule are dealt with. Some simulation experiment is undertaken, and the experiment results tell us that the expert S-plane controller is far better than the common S-plane controller.

\section{References}

[1] Li-Rong Wang. Sliding mode fault diagnosis of an underwater vehicle. Journal of Harbin Engineering University, 39, pp. 55-59, 2005.

[2] Yu-Shan Sun. Design of motion control of dam safety inspection underwater vehicle. Journal of Central South University, 19, pp. 1522-1529, 2012.

[3] Jian-Guo Wang. Improved BP algorithm applied to motion control of UV. Journal of Applied Mechanics and Materials, 446, pp. 1183-1187, 2014. 
[4] Jian-Guo Wang. An Improved S-Plane Controller for the Motion Control of Underwater Vehicles. Journal of Chinese Ship Research, 5, pp. 41-45, 2013.

[5] Yu-Shan Sun. Research on control of GDROV using adaptive fuzzy mode. Journal of The Ocean Engineering, 4, pp. 52-55, 2005.

[6] Yong Gan. Research on the motion control system of GDROV, Journal of Harbin Engineering University, 5, pp. 575-579, 2005.

[7] Lei Wan. Research on Thruster Fault Diagnosis of Underwater Vehicles Based on NN. Journal of SHIPBUILDING OF CHINA, 4, pp. 139-146, 2011.

[8] Jian-Guo Wang. Neural network identification of underwater vehicle by hybrid learning algorithm. Journal of Central South University(Science and Technology), 1, pp. 427-431, 2011.

[9] Jian-Guo Wang. System Identification of Underwater Vehicles with ML Algorithm. Journal of Applied Mechanics and Materials, 455, pp. 366-371, 2014.

[10] Yu-Shan Sun. Research on the Embedded Navigation System of AutonomousUnderwater Vehicle Based on the VxWorks. Journal of BASIC SCIENCE AND ENGINEERING, 6, pp. 899-909, 2009. 